\title{
FALSTAFF, an apparatus to study fission fragment properties
}

\section{First arm results}

Quentin Deshayes ${ }^{1, *}$, Eric Berthoumieux ${ }^{1}$, Diane Doré ${ }^{1, * *}$, Lö̈c Thulliez ${ }^{1}$, Michel Combet ${ }^{1}$, Mariam Kebbiri ${ }^{1}$, Philippe Legou $^{1}$, Alain Marcel ${ }^{1}$, Jean-Philippe Mols ${ }^{1}$, Marc-Olivier Frégeau ${ }^{2}$, Sébastien Herlant ${ }^{2}$, Xavier Ledoux ${ }^{2}$, and Julien Pancin $^{2}$

${ }^{1}$ Irfu, CEA, Université Paris-Saclay, 91191, Gif-sur-Yvette, France

${ }^{2}$ GANIL, CEA/DRF-CNRS/IN2P3, F-14076 Caen Cedex 5, France

\begin{abstract}
Nuclear fission is a complex process that still need fundamental studies. New measurements, particularly of correlated observables, could allow to develop more sophisticated theoretical models to eventually have truly predictive capabilities for the physics of fission. Moreover, the next generation reactors concepts are mostly foreseen to operate in the fast-neutron energy domain, requiring new high quality nuclear data. In this context, a new experimental setup, called FALSTAFF, dedicated to the study of fission is under development. The FALSTAFF setup aims to investigate the fission of actinides in the fast-neutron energy domain (from a few hundreds of $\mathrm{keV}$ to a few $\mathrm{MeV}$ ). Once completed, this two-arm spectrometer will detect both fragments in coincidence and allow to measure their time of flight (ToF) and kinetic energy. The average neutron multiplicity as a function of the fission fragment mass can then be assessed.

The first arm of the FALSTAFF spectrometer was built. It is composed of two main parts: first, two SEDMWPC (Multi-Wire Proportional Counter) detectors are used to measure the time-of-flight as well as the position of the fragments, thus reconstructing their velocity. Second, an axial ionisation chamber gives their kinetic energy and the energy loss profile. This proceeding will describe the FALSTAFF setup as well as the methods that are used to extract the required observables, leading up to the reconstruction of the neutron multiplicity to study the fission process. Then, the recent results obtained with the first arm of FALSTAFF will be presented, exhibiting kinetic energy, velocity and post-evaporation mass distributions. These observables will be displayed for ${ }^{252} \mathrm{Cf}$ spontaneous fission and some of the improvements recently made will be discussed.
\end{abstract}

\section{Introduction}

Nuclear fission is a complex phenomenon which is still - after several decades of study - not completely understood. New measurements, particularly of correlated observables, could allow us to feed up theoretical models to develop more sophisticated models. From an application stand point, data are very scarce on the fast-neutron domain while it has been shown [1] that the evolution of neutron multiplicity as a function of the pre-evaporation mass of the fragments is sensitive to the energy of the incoming neutron for the heavy fragments. As an option for next generation reactors is to operate on the fast-energy domain, it is critical to obtain data about neutron multiplicity and fission yields to improve the reactor simulations and develop the evaluation libraries.

To tackle this problematic, a spectrometer called FALSTAFF is under development at CEA-Saclay (France) $[2,3]$. The aim of this spectrometer is to study neutroninduced fission from actinides in a neutron energy range from hundreds of $\mathrm{keV}$ to a few $\mathrm{MeV}$. When complete, it will allow to detect the two fission fragments in coincidence and measure their kinetic energies, their initial

\footnotetext{
*e-mail: quentin.deshayes@cea.fr

**e-mail: diane.dore@cea.fr
}

masses (before neutron evaporation) and their final masses thus, giving access to the neutron multiplicity. ${ }^{238,235} \mathrm{U}$, ${ }^{239} \mathrm{Pu},{ }^{237} \mathrm{~Np},{ }^{232} \mathrm{Th},{ }^{233} \mathrm{U}$ are foreseen to be studied using this device at the NFS facility [5] .

This paper will first describe the method used to reconstruct the variables of interest as well as the experimental setup. Next, we will present some of the results obtained using the first arm of the apparatus which is already constructed and conclude showing the forthcoming steps.

\section{Setup and methods}

The FALSTAFF spectrometer is meant to provide the full characterization of the fission fragments, i.e. their masses before and after neutron evaporation process, their kinetic energies and their nuclear charges. Using those information, one could deduce the neutron multiplicity as a function of mass, which will provide valuable data about the energy sharing between the two fragments at the scission point.

The mass before neutron evaporation is obtained via the 2V (Double Velocity) method. To apply this method the assumption is made that the neutron emission, in average, does not change the velocity of the fragments in 


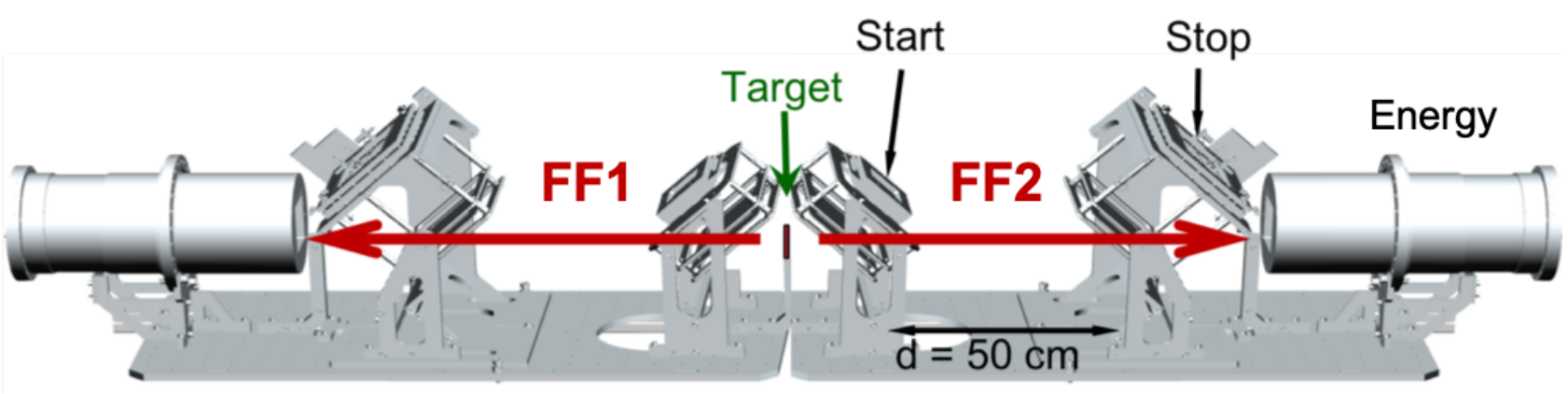

Figure 1. Drawing of the two-arm-FALSTAFF-spectrometer. Each arm is made of two ToF detectors followed by an energy detector to collect the remaining kinetic energy of the fragments. At the moment the first arm has been built.

the center of mass frame. It requires the measurement of both fragment velocities in coincidence. The velocity is determined with two time-of-flight (ToF) Secondary Electron Detectors (SeD) [4], represented in Fig. 1. Each detector gives the arrival time and position of a particle on the detector. Those detectors have a timing resolution of $\sigma_{t}=120 \mathrm{ps}$ and a spatial resolution of $\sigma_{x}=2 \mathrm{~mm}$ [6]. The distance between the two SeDs is $50 \mathrm{~cm}$. A SeD is made of an emissive foil and a Multi-Wire Proportional Chamber (MWPC) detector. When a fragment crosses an emissive foil, it loses kinetic energy leading to electron production on the foil surface. The electrons, thanks to an electric field, are then accelerated and detected by the MWPC detector.

The mass after neutron evaporation is obtained with the EV (Energy-Velocity) method. In addition to the velocity information, the kinetic energy value of the fragment is then required. This information is obtained with an axial ionisation chamber placed after the Stop detector. The kinetic energy value measured in the chamber has to be corrected for energy losses suffered by the fragment in the target, in the emissive foils and in the chamber entrance window. Those corrections require the knowledge of the fragment nuclear charge and the thicknesses of the materials the fragments have gone through. The thickness distribution of the foil is measured with an alpha transmission bench. Then the crossed thickness is deduced from this average value and the angle between the detector and the particle trajectory [7]. The nuclear charge is assumed to supply the UCD prescription [8] which suppose that the primary fission fragments have the same proton-to-mass ratio as in a fissioning nucleus. Then, an iterative procedure is applied where the energy, the mass and the charge are calculated after each correction step.

At the moment, two independent acquisition systems (DAQ) are used to collect the data: The cathode pad/strips signals from the Start and Stop detectors are digitized using the GET (General Electronics for TPCs) acquisition system [9] on a $\mu$ TCA crate, All others signals (timing information from Start and Stop detectors, anode and grid signal from the axial ionisation chamber) are recorded by using the standard GANIL acquisition based on a VME crate. For the Start and Stop detectors the anode signals are digitized by using a Matacq card [10] having a $2 \mathrm{GHz}$ sampling rate. The anode and grid signals from the ionisation chamber are digitized by using a CAEN V1724 card having $100 \mathrm{MHz}$ sampling rate.

\section{Results}

In 2018 the full setup was installed in a dedicated reaction chamber. A ${ }^{252} \mathrm{Cf}$ source was placed at the target position, using a collimator to limite the angular opening to \pm 3 deg. Results were encouraging but the specifications of the apparatus were not yet fulfilled [11]. Some improvements have been added to the setup in early 2019 and in this section, we will describe the results that we obtained and compare them to simulation results. The simulation was using the GEANT4 framework (version 10-02) [12] and the fission observables were determined using the GEF code [13]. The spatial and timing resolutions used in the simulation are $\sigma_{x}=2 \mathrm{~mm}$ and $\sigma_{t}=120 \mathrm{ps}$. In the Fig. 2, 3, 4 and 5, the experimental data are presented in red while the simulation results are in dashed-blue. On all those figures, data are normalized to their integral.

The LoF (Length-of-Flight) is calculated from the positions measured in the Start and Stop detectors. The angle between the track of the fragments and the emissive foils is also calculated providing the thickness crossed by the fragments. This information is needed to calculate the energy loss by the fragments. The left panel of Fig. 2 shows the comparison between experimental and simulated LoF distributions. One can observe that the simulation has the good angular parameters since the LoF is well reproduced.

One of the improvements made in 2019 was the replacement of the Start detector as the previous one was too noisy which resulted in the inability to distinguish the less energetic events from the electronic noise. As the heavy fission fragments deposit less energy in matter than the light ones, this resulted in an unbalance in the number of fragments detected which was detrimental to all the other observables. The difference between the time of arrival of the fragments at the stop and the new Start detector is presented in Fig.2 (right pannel). The noise issue was solved and the balance between light and heavy fragments restored. The time was calibrated thanks to the measurement with an alpha source and the agreement with the simulations data is excellent. 


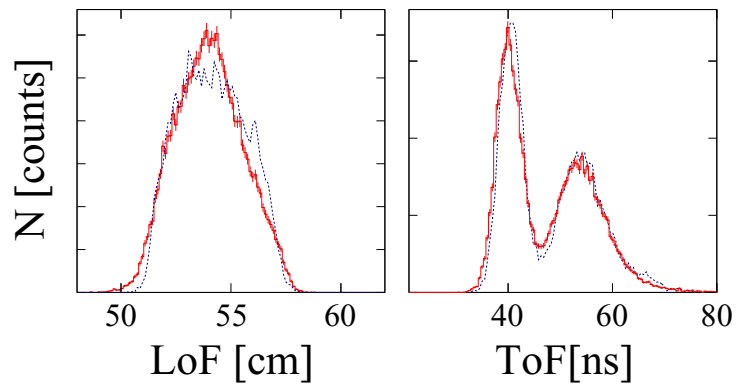

Figure 2. Distributions of the LoF (left) and the ToF (right) of fragments issued from spontaneous fission of a ${ }^{252} \mathrm{Cf}$ source. The red line is the data measured with the FALSTAFF setup while the blue line is the result of a GEANT4 simulation of the setup using GEF.

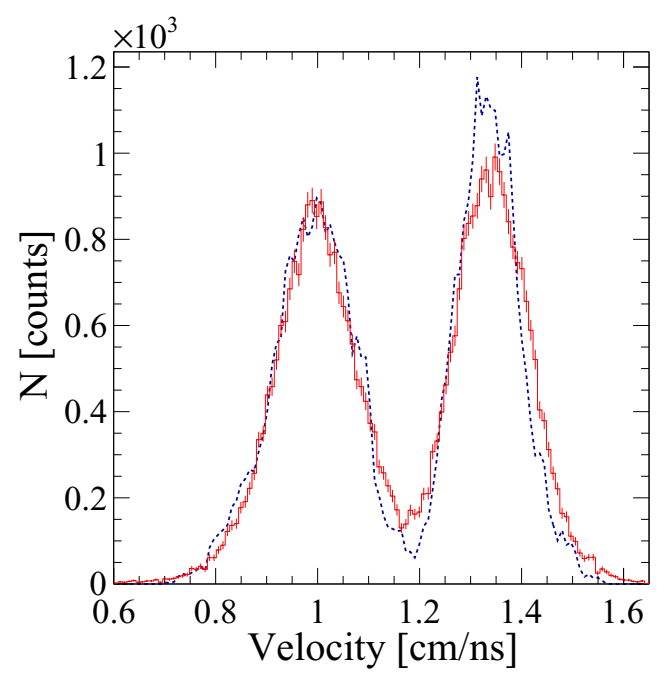

Figure 3. Distributions of velocity of fragments issued from spontaneous fission of a ${ }^{252} \mathrm{Cf}$ source. The red line is the data measured with the FALSTAFF setup while the blue line is the result of a GEANT4 simulation of the setup using GEF.

Based on the ToF and LoF measurements, the velocity is calculated event-by-event. Fig. 3 shows the experimental (red curve) and the simulated (G4-GEF (blue curve)) distributions. Agreement between simulation and experiment is very good although the resolution for the light fragments seems slightly worse in the experiment.

The kinetic energy distribution is presented in Fig. 4, after having been corrected from the energy lost in the entrance window of the ionisation chamber and the emissive foil of the Stop detector. The calibration procedure is based on data and GEANT4 tables. In order to perform this calibration, the $\mathrm{Cf}$ source was placed at three different positions: target position, between Start and Stop detector and in front of the ionisation chamber.

Due to the low energy of fission fragments (60$100 \mathrm{MeV}$ ), energy losses in the materials are important. The kinetic energy measured in the chamber has to be corrected for energy losses suffered by the fragment in the emissive foil and in the ionisation chamber entrance window. One obtains the energy at the point where the veloc-

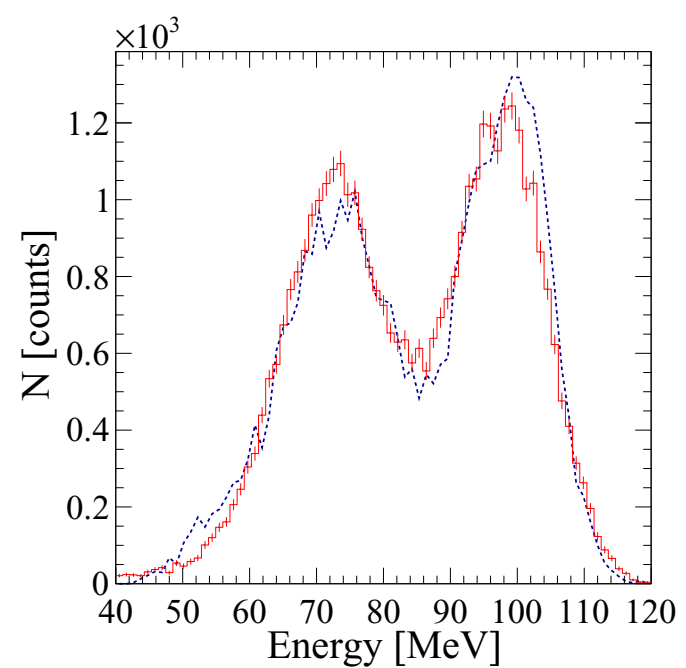

Figure 4. Distributions of kinetic energy of fragments issued from spontaneous fission of a ${ }^{252} \mathrm{Cf}$ source, reconstructed between the two ToF detectors. The red line is the data measured with the FALSTAFF setup while the blue line is the result of a GEANT4 simulation of the setup using GEF.

ity is measured. One can then apply the method described in Section 2 to obtain the mass distribution of the fission fragments after neutron evaporation.

The experimental and simulated distributions obtained for the fragment mass after the iterative procedure are shown in Fig. 5. They both go through the same analysis process, starting from the residual energy detected in the ionisation chamber which is corrected up to the point between the two ToF detectors. The main difference is the width of the experimental distribution for heavy fragments which is higher in the experimental data. The discrepancy for the width could be due to the energy resolution or the uncertainty on the energy loss by the fission fragments in matter.

\section{Conclusion and Outlooks}

The results showed in the previous section are very encouraging. Yet, the resolution on the reconstruction of the postneutron-evaporation mass still needs to be improved. One of the key point to do so, is to ensure that one does have a good knowledge of the energy loss of the fission fragments inside matter. Unfortunately, up to this point, there are very few measurements of energy loss of heavy ions in composite material at the energy range of interest (inferior to $100 \mathrm{MeV}$ ) [14]. Moreover, if one compares the energy loss of ions in a foil of $0.9 \mu \mathrm{m}$ of mylar (typical width of the entrance window of the ionisation chamber) calculated with different codes such as SRIM [15], LISE++ [16] and the GEANT4 prescriptions EMZ/EMV, one can observe discrepancies as high as $3 \mathrm{MeV}$ for heavy fragments like ${ }^{140} \mathrm{Cs}$. The effect of the choice of GEANT4 prescription on the post-neutron-evaporation mass is showed in Fig. 6. To try to select the best possible model for the energy loss, a dedicated experiment will be held at the Institut Laue 


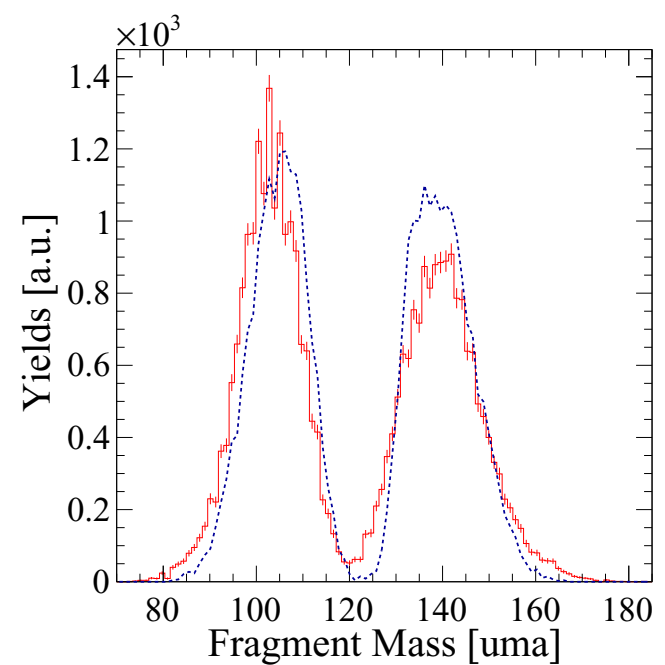

Figure 5. Distributions of post-evaporation masses of fragments issued from spontaneous fission of a ${ }^{252} \mathrm{Cf}$ source. The red line is the data measured with the FALSTAFF setup while the blue line is the result of a GEANT4 simulation of the setup using GEF.

Langevin (ILL) (France) in September 2019. The Lohengrin setup will be used to perform energy loss measurements on a large variety of fission fragments at different energies that will pass through different materials.

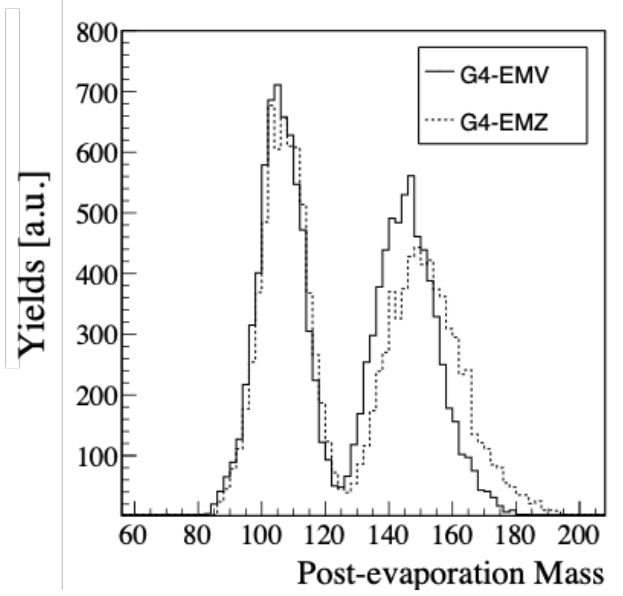

Figure 6. Post neutron evaporation mass of the fission fragments of a ${ }^{252} \mathrm{Cf}$ source reconstructed using two different prescription of Geant4.
One could also consider to couple the FALSTAFF device to some Germanium detectors to study the structure of fission fragments by measuring triple coincidences between one fragment and two gamma particles. One of the fragments would be detected in FALSTAFF while the other one would be stopped to avoid the Doppler effect. This type of experiment would allow a direct comparison with fission and deexcitation models and indirectly to get information on the angular momentum of the fission fragment at scission.

\section{Acknowledgement}

This work was partially supported by CEA, NEEDS and within the Seventh Frame-work Programme through CHANDA (EURATOM contract no. FP7-605203). The authors would like to specially thank the P2IO LabEx (ANR-10-LABX-0038) in the framework "Investissements d'Avenir" (ANR-11-IDEX-0003-01) managed by the Agence Nationale de la Recherche (ANR, France) for making this work possible.

\section{References}

[1] A. A. Naqvi et al., Phys. Rev. C 34, 218 (1986)

[2] D. Doré et al., Nuclear Data Sheets 119, 346 (2014)

[3] L. Thulliez PhD. Thesis, Université Paris Saclay (2017)

[4] A. Drouart et al., Nucl. Instr. Meth. Phys. Res. A 506, 250 (2003)

[5] X. Ledoux et al., Nuclear Data Sheets 119, 353 (2014)

[6] M. Vostinar et al., JINST 119 (2013)

[7] L. Thulliez et al., EPJ Web Conf. 146, 04028 (2017)

[8] A. C. Wahl. Atomic Data and Nuclear Data Tables, 39, 156 (1988)

[9] E. Pollaco et al., Nucl. Instr. Meth Phys. Res. A, 887, 81 (2018)

[10] D. Breton and E. Delagnes, IEEE NSS 2006, San Diego, USA (2006)

[11] D. Doré et al., EPJ Web Conf. 211, 04002 (2019)

[12] S. Agostinelli et al., Nucl. Instr. Meth. Phys. Res. A 506, 250

[13] K. H. Schmidt et al., JEFF Report 24, (2014)

[14] K. Wittmaack, Nucl. Instr. Meth., B380, 57 (2016)

[15] J.F. Ziegler et al., SRIM code available from http:/www.srim.org

[16] LISE++ software, http://lise.nscl.msu.edu/lise.html 